\title{
Neuroprotection by (-)-epigallocatechin-3-gallate in a rat model of stroke is mediated through inhibition of endoplasmic reticulum stress
}

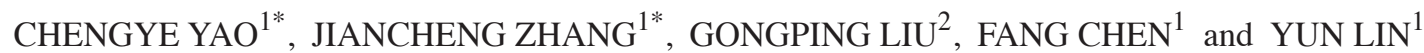 \\ ${ }^{1}$ Department of Anesthesiology, Union Hospital; ${ }^{2}$ Department of Pathophysiology, Tongji Medical \\ College, Huazhong University of Science and Technology, Wuhan, Hubei 430022, P.R. China
}

Received March 3, 2013; Accepted October 25, 2013

DOI: $10.3892 / \mathrm{mmr} .2013 .1778$

\begin{abstract}
Epigallocatechin-3-gallate (EGCG), the predominant constituent of green tea, has been demonstrated to be neuroprotective against stroke in rats. However, the precise mechanism of EGCG responsible for neuroprotective activity remains unclear and no established treatment for decreasing the resulting neurological damage of stroke exists. The present study was designed to investigate the neuroprotective mechanism of EGCG on transient focal cerebral ischemia in rats. EGCG, when applied immediately following ischemia, significantly decreased the expression of endoplasmic reticulum stress (ERS)-related markers, [glucose-regulated protein 78 (GRP78), C/EBP-homologous protein (CHOP) and caspase-12] and apoptosis $24 \mathrm{~h}$ following reperfusion. EGCG treatment also significantly reduced infarct volumes and increased neurological scores which was correlated with elevated levels of TRPC6 and phosphorylation of $\mathrm{cAMP} / \mathrm{Ca}^{2+}$ response element-binding protein (p-CREB) activity, and decreased calpain-specific aII-spectrin breakdown product (SBDP145) activity. When mitogen-activated protein kinase kinase (MEK) activity was specifically inhibited, the neuroprotective effect of EGCG was attenuated and a correlated decrease in CREB activity was observed. In conclusion, the results clearly demonstrated that intracerebroventricular injection of EGCG immediately following ischemia, inhibits ERS and improves the neurological status of rats that have undergone middle cerebral artery occlusion via the inhibition of calpain-mediated TRPC6
\end{abstract}

Correspondence to: Dr Yun Lin, Department of Anesthesiology, Union Hospital, Tongji Medical College, Huazhong University of Science and Technology, 1277 Jiefang Avenue, Wuhan, Hubei 430022, P.R. China

E-mail: linyun7801@yahoo.com.cn

${ }^{*}$ Contributed equally

Key words: $\mathrm{cAMP} / \mathrm{Ca}^{2+}$ response element-binding protein, (-)-Epigallocatechin-3-gallate, endoplasmic reticulum stress, middle cerebral artery occlusion, TrpC proteolysis and the subsequent activation of CREB via the MEK/extracellular signal-regulated kinases (ERK) pathway.

\section{Introduction}

The endoplasmic reticulum (ER) is an organelle that folds and synthesizes transmembrane, intraorganellar and secretory proteins. The disequilibrium of ER homeostasis, including glucose deprivation, disturbance of the redox environment, perturbation of calcium homeostasis and exposure to free radicals disrupts the normal function of the ER and induces ER stress (1). Pro-apoptotic proteins are expressed under ER stress, including CHOP and caspase- 12 and -3 , which results in neuronal cell death. ER stress plays a role in the pathogenesis of a variety of human diseases, including neuronal degenerative diseases, ischemia/reperfusion injury, and heart diseases (2-4).

The transient receptor potential cation channels (TRPC) are a subfamily of nonselective cation channels permeable to $\mathrm{Ca}^{2+}$, which are present in numerous cell types including neurons $(5,6)$. The TRPC6 channel is involved in the promotion of neuronal survival following focal cerebral ischemia. Activation of calpain leads to TRPC6 degradation and neuronal damage in ischemia (7). Previous studies have determined that the TRPC6 channel is essential in promoting neuronal survival and indicates that the activation of CREB is a key downstream effector for the neuronal protective effect of the TRPC6 channel in vitro and in vivo $(7,8)$. Therefore, it may be a used as a novel therapeutic strategy to protect against ischemic brain damage as the inhibition of TRPC6 degradation preserves neuronal survival.

EGCG is the predominant constituent of green tea (9). It has been shown to promote neuronal plasticity (10) and to improve cognitive function and learning ability $(11,12)$. In addition, EGCG has also been demonstrated to reduce delayed cell death near the hippocampus and the excitotoxic neuronal damage that occurs in ischemic lesions following transient ischemia $(2,13,14)$. However, the precise mechanism of the neuroprotective activity of EGCG remains unclear. Previous studies have not fully demonstrated the effects of EGCG on TRPC6/CREB-mediated neuroprotection.

The aim of this study was to investigate whether the administration of EGCG immediately following ischemia exhibits 
A

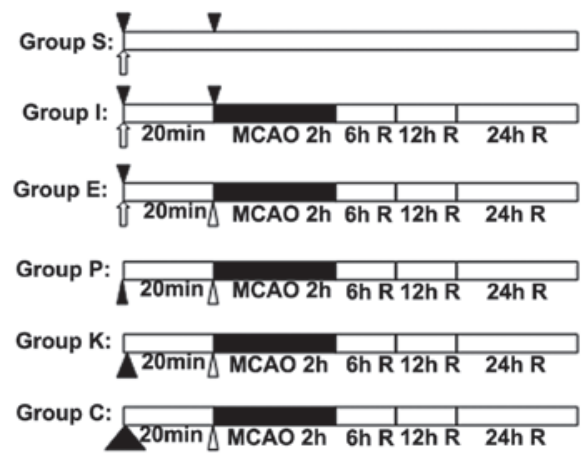

B

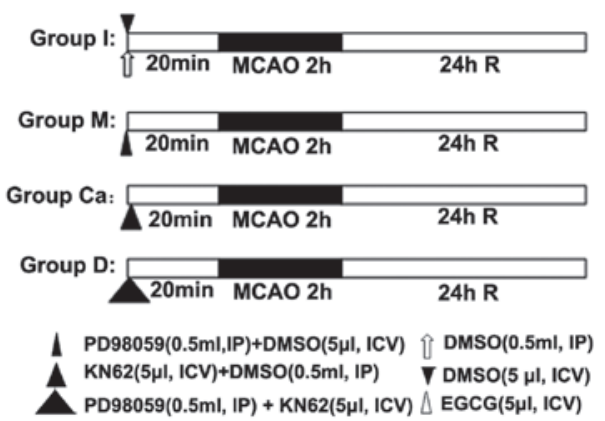

C

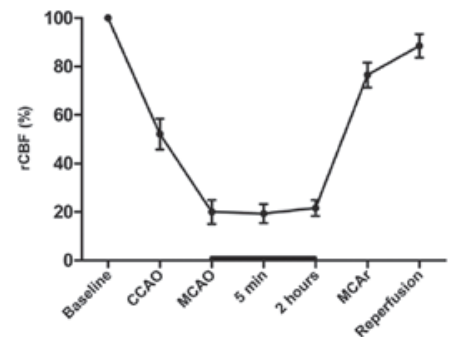

D

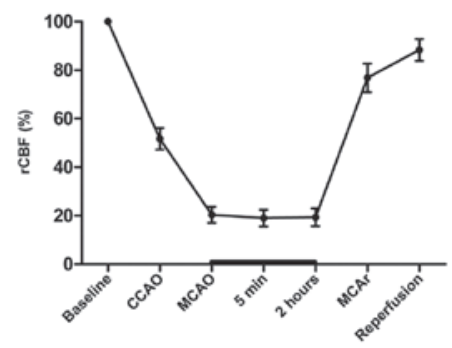

Figure 1. Experimental protocol. (A) Rats were randomly divided into 6 groups, and each group was again divided into 3 subgroups according to the time of reperfusion following ischemia (6, 12 and $24 \mathrm{~h}$ following reperfusion) ( $\mathrm{n}=12$ per subgroup). (C) Another 45 rats were randomly divided into four groups. (B and D) Change in regional cerebral blood flow (rCBF) in rats. Right common carotid artery (CCA) occlusion reduced CBF to $\sim 50 \%$ of the baseline, and additional middle cerebral artery (MCA) occlusion further decreased rCBF to 20\%. Group S (subgroup S6, S12 and S24), Sham-operation; group I (subgroup I6, I12 and I24), middle cerebral artery occlusion (MCAO); group E (subgroup E6, E12 and E24), ischemia combined with EGCG treatment; Group P (subgroup P6, P12 and P24), ischemia combined with EGCG plus PD98059 treatment; Group K (subgroup K6, K12 and K24), ischemia combined with EGCG plus KN62 treatment; group C (subgroup C6, C12 and C24), ischemia combined with EGCG plus PD98059 and KN62 treatment; group M, ischemia combined with PD98059 treatment; Group Ca: ischemia combined with KN62 treatment; Group D: ischemia combined with PD98059 plus KN62 treatment. R, reperfusion; CCAO, CCA occlusion; MCAO, MCA occlusion; MCAR, MCA remove.

a neuroprotective effect on ischemic neurons in a middle cerebral artery occlusion (MCAO) rat model. This study also aimed to determine whether EGCG inhibits ER stress (ERS) and improves the neurological status through the inhibition of calpain-mediated TRPC6 proteolysis and the subsequent activation of CREB via the mitogen-activated protein kinase kinase (MEK)/extracellular signal-regulated kinases (ERK) pathway.

\section{Materials and methods}

Middle cerebral artery occlusion (MCAO) model. Male Sprague-Dawley rats (weight, 200-250 g) were purchased from Hunan Weasleyg Scene of Experimental Animals Co., Ltd. (Hubei, China). The experiments were approved by the Committee of Experimental Animals of Tongji Medical College (Hubei, China) and conformed to internationally accepted ethical standards (Guide for the care and use of laboratory animals; NIH Publication 80-23, revised 1978). Briefly, rats were anesthetized with an intraperitoneal (i.p.) injection of chloral hydrate $(400 \mathrm{mg} / \mathrm{kg})$ and placed in the supine position with the limbs taped to the operation table. A midline skin incision was performed, the right external carotid artery was exposed and its branches were ligated. A 4-0 monofilament nylon suture (Beijing Shandong Industrial Corp., Beijing, China) with a rounded tip was introduced into the internal carotid artery through the common carotid artery and advanced until faint resistance was felt. Following $2 \mathrm{~h}$ of transient MCAO, blood flow was restored by the withdrawal of the nylon thread to allow reperfusion, which was confirmed by a laser Doppler flowmeter; Periflux system 5000, Perimed, Stockholm, Sweden. Sham-operated rats underwent the same procedure without the filament insertion. Throughout the experiments, body temperature was maintained at $37 \pm 0.5^{\circ} \mathrm{C}$ with a homeothermic (RWD Life Science Co., Ltd., Shenzhen, China). Regional cerebral blood flow (rCBF) was monitored by a laser-Doppler flowmeter prior to, during and following MCAO, as well as prior to death (Fig. 1). Animals that did not show a $\mathrm{CBF}$ reduction of $\geq 70 \%$ and animals that died following ischemia induction were excluded from the experimental group. Prior to reperfusion, rats with incomplete MCAO ( 10\%) were excluded from further study by a blinded observer.

Drug treatment. Rat intracerebroventricular (ICV) injection was performed under anesthesia using a stereotaxic instrument (RWD Life Science Co., Ltd.) with a microsyringe pump (Shanghai Guangzheng Medical Equipment Co., Ltd., Shanghai, China). A scalp incision was perfomed and a burr hole was made in the right parietal skull, $1.8 \mathrm{~mm}$ lateral and $1.0 \mathrm{~mm}$ posterior to the bregma. A syringe was inserted into the brain to a depth of $4.2 \mathrm{~mm}$ below the cortical surface. EGCG or PD98059 was dissolved in dimethyl sulfoxide (DMSO) (all obtained from Sigma-Aldrich, St. Louis, MO, USA). EGCG $(1 \mathrm{mg} / \mathrm{ml} ; 5 \mu \mathrm{l})$ or $1 \%$ DMSO $(5 \mu \mathrm{l})$ was injected slowly $(0.5 \mu \mathrm{l} / \mathrm{min})$ into the right ventricle immediately following 
ischemia. PD98059 (0.75 mg/rat, i.p.) or $1 \%$ DMSO (0.5 ml, i.p.) was administered to rats 20 min prior to the operation.

The rats were randomly divided into four groups and each group was again divided into three subgroups ( $n=12$ per subgroup) according to the time of reperfusion following ischemia. The experimental groups and subgroups were as follows: Sham-operation (group S; subgroup S6, S12 and S24); MCAO (group I; subgroup I6, I12 and I24); ischemia combined with EGCG treatment (group E; subgroup E6, E12 and E24) and ischemia combined with EGCG plus PD98059 [a mitogen-activated protein kinase kinase (MEK) inhibitor] treatment (group C; subgroup C6, C12 and C24). Another 27 rats were randomly divided into 3 groups ( $\mathrm{n}=9$ per group): Sham-operation; MCAO (Group I) and ischemia combined with PD98059 treatment (Group P).

Measurements of infarct volume. At $24 \mathrm{~h}$ following reperfusion, rats were decapitated and the brains were rapidly removed and frozen at $-20^{\circ} \mathrm{C}$ for $10 \mathrm{~min}$. Sliced brain tissues were stained with $2 \%$ 2,3,5-triphenyltetrazolium chloride (TTC; Sigma-Aldrich) for $30 \mathrm{~min}$ at $37^{\circ} \mathrm{C}$ followed by overnight immersion in $4 \%$ paraformaldehyde. The extent of ischemic infarction was traced and the integrated volume was calculated using ImageJ 1.45 software (National Institutes of Health, Bethesda, MD, USA). The relative infarction volume was calculated by the following equation, giving a correction for edema: \{[Total lesion volume - (ipsilateral hemisphere volume - contralateral hemisphere volume)] / contralateral hemisphere volume\} x100.

Neurological scoring. Neurological scores were evaluated by a blinded observer $24 \mathrm{~h}$ following reperfusion with a scoring system as described previously $(15,16)$.

Western blot analysis. The rats were euthanized by decapitation at 6,12 and $24 \mathrm{~h}$ following reperfusion and the infarct side of the cortex was harvested. Total protein extraction was performed according to the manufacturer's instructions in the kit (KGP250; Keygen Biotech, Nanjing, China) for western blot analysis of TRPC6 and aII-spectrin. Nuclear protein extraction was performed according to the manufacturer's instructions in the kit (Fisher Scientific, Pittsburgh, PA, USA) for p-CREB. Protein levels in the extracts were quantified using a bicinchoninic acid (BCA) assay. Equal quantities of total or nuclear protein extracts were separated by sodium dodecyl sulphate polyacrylamide gel electrophoresis and transferred to polyvinylidene difluoride membranes by electrophoresis. Membranes were incubated in Tris-buffered saline, TBS containing $1 \%$ Tween-20 (TBST) blocking buffer and 5\% non-fat dry milk for $1 \mathrm{~h}$ at room temperature. Membranes were incubated overnight at $4^{\circ} \mathrm{C}$ with either a mouse monoclonal anti-aII-spectrin (dilution, 1:1000; Enzo Biochem, New York, NY, USA), rabbit polyclonal anti-TRPC6 (dilution, 1:1000; Abcam Cambridge, MA, USA), rabbit monoclonal anti-p-CREB (dilution, 1:1000) mouse monoclonal anti-CHOP (dilution, 1:1000), rabbit polyclonal anti-GRP78 (dilution, 1:1000; Cell Signaling Technology Inc., Beverly, MA, USA), rabbit polyclonal anti-caspase-12 (dilution, 1:200; Beijing Biosynthesis Biotechnology Co., Ltd, Beijing, China), rabbit polyclonal anti-Lamin B1 (dilution, 1:500; Bioworld Technology Inc., Harrogate, UK) or mouse monoclonal anti-glyceraldehyde 3-phosphate dehydrogenase (GAPDH) antibody (dilution, 1:1000; Proteintech Group, Inc., Hubei, China). This was followed by incubation with horseradish peroxidase-labeled secondary anti-mouse $\operatorname{IgG}$ or anti-rabbit IgG antibodies (dilution, 1:5000; Proteintech Group, Inc.), respectively. Labeled proteins were detected with the ChemiDocXRS ${ }^{+}$chemiluminescence imaging system (Bio-Rad, Hercules, CA, USA) and bands were quantified using lab imaging software. The experiments were repeated in triplicate.

Quantum dot-based immunofluorescence and immunohistochemistry. At $24 \mathrm{~h}$ following reperfusion, the rats were perfused with $250 \mathrm{ml}$ of $0.9 \%$ cold saline followed by $100 \mathrm{ml}$ of $4 \%$ paraformaldehyde in phosphate-buffered saline ( $\mathrm{pH}$ 7.4). The brains were then rapidly removed, blocked and embedded in paraffin. Paraffin-embedded brains were cut into 4- $\mu \mathrm{m}$ thick sections according to standard procedures. The paraffin sections ( $\mathrm{n}=3$ for each group) were incubated overnight with antibodies against TRPC6 (1:100; Abcam) at $4^{\circ} \mathrm{C}$ subsequent to blocking with bovine serum albumin (BSA). The samples were then incubated with a biotinylated secondary antibody at $37^{\circ} \mathrm{C}$ for $30 \mathrm{~min}$. Subsequent to blocking with BSA, the paraffin sections were incubated with streptavidin-conjugated QDs605 (dilution, 1:100; Wuhan Jiayuan Quantum Dots Co., Ltd., Hubei, China). The cell nuclei were stained with 4',6-diamidino2-phenylindole (DAPI). TRPC6-positive cells were measured at a magnification of $x 200$ per visual field in the peri-infarct region; three visual fields per section and three brain sections per rat were analyzed. Fluorescent signals were detected by fluorescence microscopy (BX51; Olympus, Tokyo, Japan) and signal intensities were collected for statistical analysis. Images were captured with a Doppler imaging system (CRi Nuance Fx; Caliper Life Sciences, Hopkinton, MA, USA).

TdT mediated dUTP nick-end labeling (TUNEL) assay. TUNEL staining analysis was used to detect apoptotic cell death $24 \mathrm{~h}$ following reperfusion. TUNEL staining was conducted with a kit (Roche Diagnostics GmbH, Mannheim, Germany). TUNEL-positive nuclei with chromatin condensation and fragmented nuclei were considered as probable apoptotic cells. The total number of TUNEL-positive neurons in the ipsilateral hemisphere was counted in three different fields for each section (by an investigator who was blinded to the studies) by light microscopy at a magnification of $x 400$.

Statistical analysis. GraphPad Prism software (version 5 for Windows, GraphPad software Inc., La Jolla, CA, USA) was used for all statistical analyses. Values are presented as the mean \pm standard error of the mean. The neurological score data comparison was analyzed using the Kruskal-Wallis test followed by a post hoc Dunn's test. For all other measurements, one-way analysis of variance followed by Newman-Keuls multiple comparison test was used. $\mathrm{P}<0.05$ was considered to indicate a statistically significant difference.

\section{Results}

EGCGblocks calpain-specific aII-spectrinbreakdown product (SBDP145) formation. Compared with the sham-operated 
A

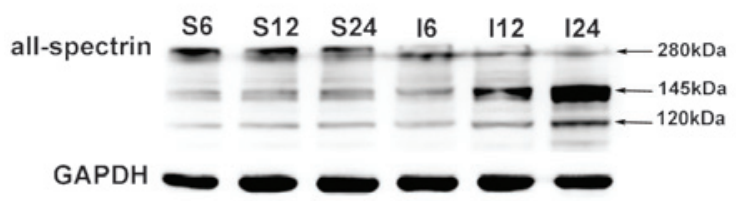

C

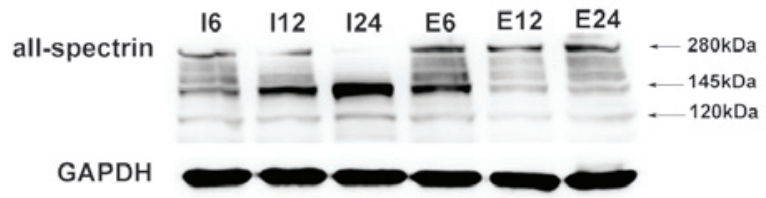

$\mathbf{E}$

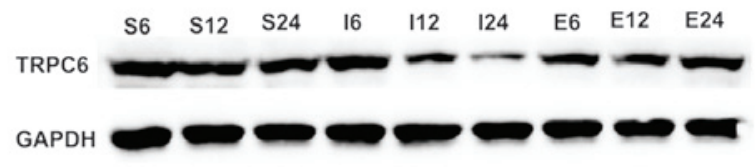

B

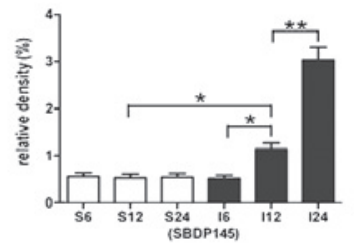

D

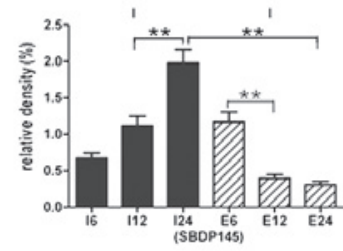

$\mathbf{F}$

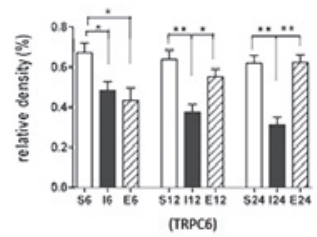

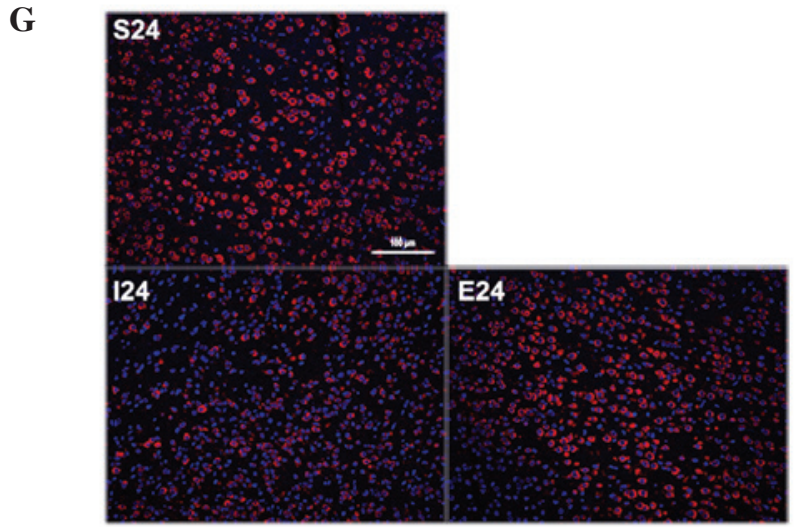

Figure 2. Effect of (-)-epigallocatechin-3-gallate (EGCG) treatment on the levels of SBDP145 and transient receptor potential cation channel (TRPC6). (A and C) Western blot analysis of SBDP145 expression in the ipsilateral cortex at 6,12 and $24 \mathrm{~h}$ following reperfusion. Glyceraldehyde 3-phosphate dehydrogenase (GAPDH) was used as a loading control. (B and D) Densitometric analysis of the protein levels of SBDP145 in the ipsilateral cortex at the indicated time points in the two groups or with sham operation $(\mathrm{n}=3)$. (E) Western blot analysis of TRPC6 expression at 6,12 and $24 \mathrm{~h}$ following reperfusion. The samples were prepared from the ipsilateral cortex. GAPDH was used as a loading control. (F) Densitometric analysis of the protein levels of TRPC6 in the ipsilateral cortex at the indicated times in the two groups or with sham operation $(n=3)$. (G) TRPC6 immunoreactivity (magnification, $x 200)(n=3)$. The nuclei are counterstained with 4',6-diamidino-2-phenylindole (DAPI). Staining was present within the cell membrane. Group S, sham-operation group; group I, middle cerebral artery occlusion (MCAO) group; group E, MCAO treated with EGCG; group C, MCAO treated with EGCG and PD98059. Values are presented as the mean \pm SEM. ${ }^{*} \mathrm{P}<0.05$ and ${ }^{* *} \mathrm{P}<0.01$.

group the SBDP145 level in the MCAO group was significantly increased after $12 \mathrm{~h}(\mathrm{P}<0.05)$, an increase that was greatest at $24 \mathrm{~h}(\mathrm{P}<0.01$; Fig. 2A and B). When MCAO rats were treated with EGCG the SBDP145 level was significantly decreased at $12 \mathrm{~h}(\mathrm{P}<0.01)$ and the decrease was greatest at $24 \mathrm{~h}(\mathrm{P}<0.01$; Fig. 2C and D).

EGCG inhibits calpain-mediated TRPC6 channel degradation. Compared with the sham-operated group, TRPC6 levels in the MCAO group were significantly decreased at 6 $(\mathrm{P}<0.05), 12$ and $24 \mathrm{~h}(\mathrm{P}<0.01$; Fig. 2E and F). When MCAO rats were treated with EGCG, the protein level of TRPC6 was significantly increased at 12 and $24 \mathrm{~h}(\mathrm{P}<0.05$ and $\mathrm{P}<0.01$, respectively). Immunofluorescence analysis showed a cytomembrane staining pattern of TRPC6 in neurons of the cerebral cortex (Fig. 2G).
PD98059 exhibits no effect on ischemic stroke in rats at $24 \mathrm{~h}$ following reperfusion. To determine the effect of PD98059 in the stroke rats, PD98059 was administered 20 min prior to the operation. Notably, with the application of PD98059 alone, no statistical significance was identified in the protein levels of p-CREB in MCAO rats (Fig. 3A). There was no significant difference between the two groups in the measurements of the the infarct volumes and neurological scores (Fig. 3B and C).

EGCG maintains phosphorylation of CREB by blocking TRPC6 degradation. Compared with the sham-operated group, the level of p-CREB in the MCAO group was significantly decreased at 12 and $24 \mathrm{~h}(\mathrm{P}<0.05$ and $\mathrm{P}<0.01$, respectively; Fig. 4A and B). Compared with the MCAO group, the level of p-CREB in the EGCG-treated group was significantly increased at 12 and $24 \mathrm{~h}(\mathrm{P}<0.05$ and $\mathrm{P}<0.01$, respectively). 
A
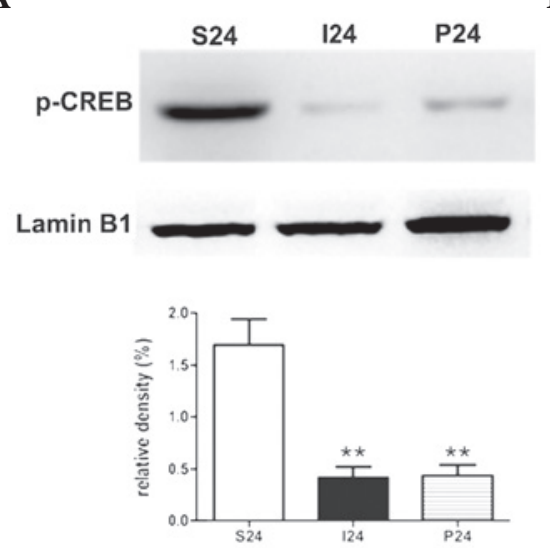

B

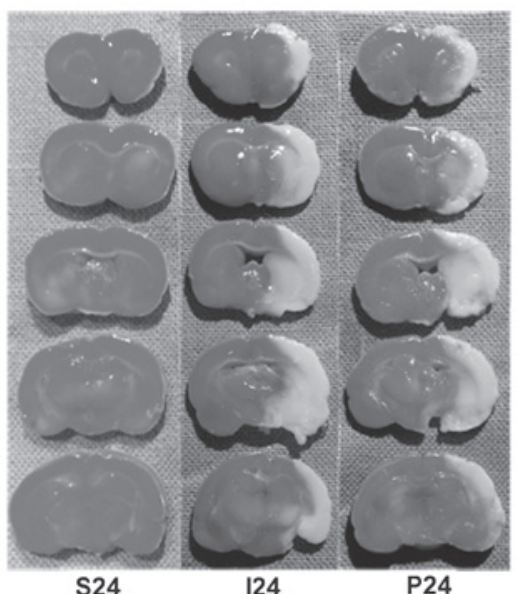

S24

124

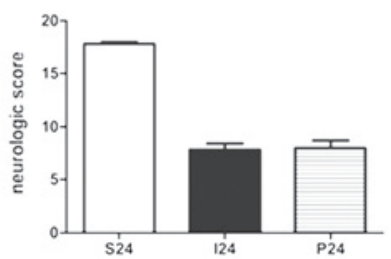

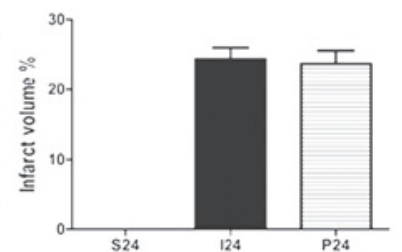

$\mathbf{C}$

Figure 3. Effect of PD98059 on the stroke rats $24 \mathrm{~h}$ following reperfusion. (A) Western blot analysis of phosphorylation of cAMP/Ca ${ }^{2+}$ response element-binding protein ( $\mathrm{p}-\mathrm{CREB})(\mathrm{n}=3)$. Nuclear protein extracts were prepared from the ipsilateral cortex. Quantification of $\mathrm{p}-\mathrm{CREB}$ assessed by western blot analysis was normalized to the expression level of Lamin B1. (B) Representative 2,3,5-triphenyltetrazolium chloride (TTC) staining of the cerebral infarct in the rat brain $(n=6)$. (C) Quantification of neurologic scores $(n=6)$. Group S, sham-operated group; group I, middle cerebral artery occlusion (MCAO) group; group P, MCAO treated with PD98059 group. Values are presented as the mean \pm SEM. ${ }^{* *} \mathrm{P}<0.01$ vs. sham-operated group.

A

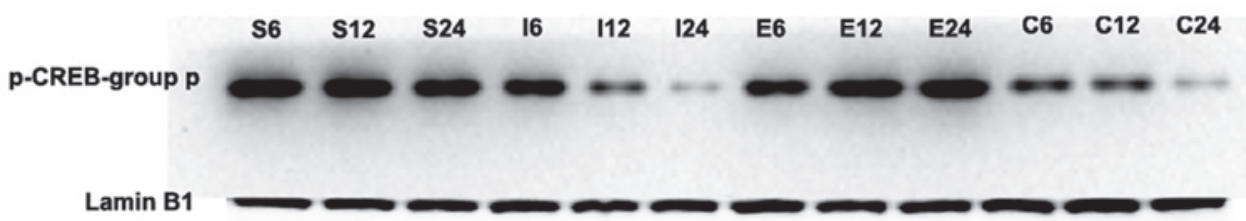

B

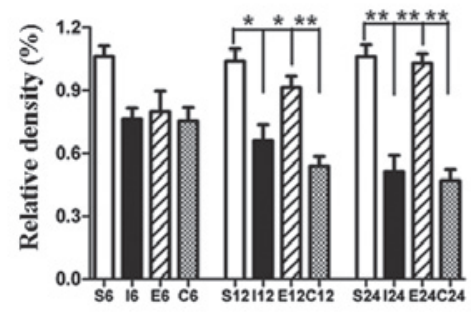

Figure 4. Effect of (-)-epigallocatechin-3-gallate (EGCG) treatment on the level of phosphorylation of cAMP/Ca ${ }^{2+}$ response element-binding protein (p-CREB), (A) Western blot analysis of p-CREB at 6,12 and $24 \mathrm{~h}$ following reperfusion. Samples were prepared from the ipsilateral cortex. Lamin B1 was used as a loading control. (B) Densitometric analysis of the protein levels of p-CREB in the ipsilateral cortex at the indicated times in the three groups or with sham operation ( $\mathrm{n}=3$ ). Group S, sham-operated group; group I, middle cerebral artery occlusion (MCAO) group; group E, MCAO treated with EGCG group; group $\mathrm{C}, \mathrm{MCAO}$ treated with EGCG and PD98059 group. Values are presented as the mean $\pm \mathrm{SEM}$. ${ }^{*} \mathrm{P}<0.05$ and ${ }^{* *} \mathrm{P}<0.01$.

In the rats treated with PD98059, the level of $\mathrm{p}-\mathrm{CREB}$ was significantly decreased at 12 and $24 \mathrm{~h}(\mathrm{P}<0.01$ and $\mathrm{P}<0.01$, respectively) compared with that of the EGCG-treated group.

EGCG inhibits ERS and apoptosis 24 following reperfusion. In the MCAO group, the protein levels of ERS-related markers GRP78, CHOP and caspase-12 were significantly increased compared with that in the sham-operation group $(\mathrm{P}<0.01$, $\mathrm{P}<0.01$ and $\mathrm{P}<0.01$, respectively; Fig. 5Aa-c). When MCAO rats were treated with EGCG, the protein levels of CHOP, GRP78 and caspase-12 were significantly decreased $(\mathrm{P}<0.01, \mathrm{P}<0.01$ and $\mathrm{P}<0.01$, respectively). Subsequent to the administration of PD98059, the protein levels were significantly increased compared with that of the EGCG-treated group.

In the TUNEL assay, EGCG significantly reduced apoptotic cell death in the right cortex compared with that in the MCAO group $(\mathrm{P}<0.01$; Fig. 5D and E). Following treatment with PD98059, the apoptotic cell death was significantly increased compared with that of the EGCG-treated group $(\mathrm{P}<0.01)$. 
A

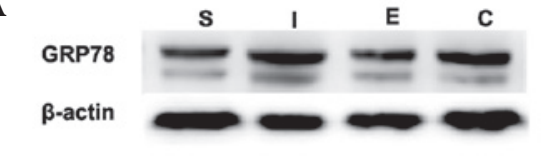

B

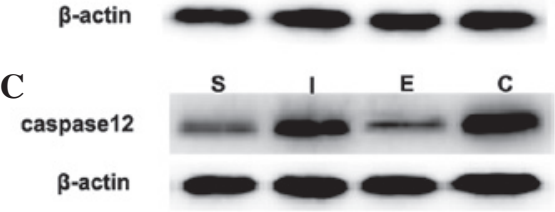

D

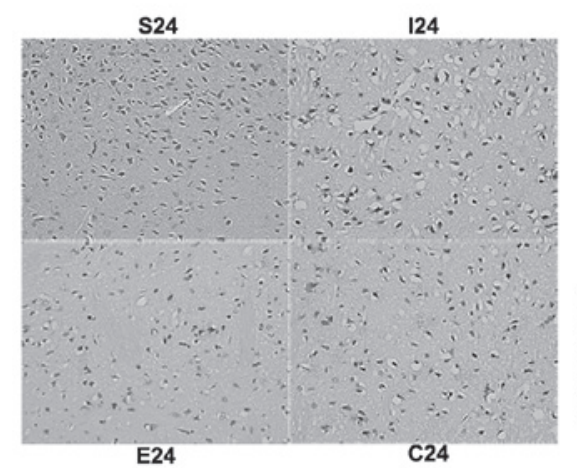

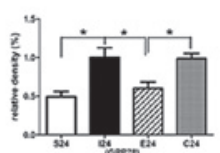
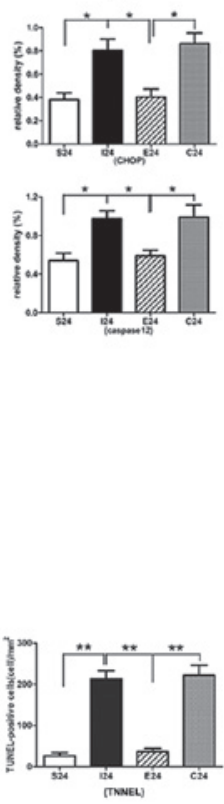

$\mathbf{E}$

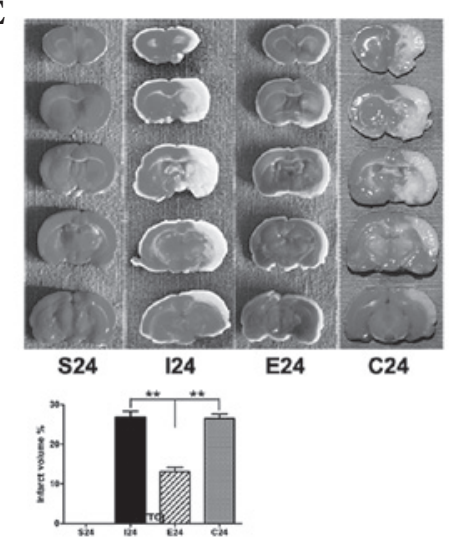

F

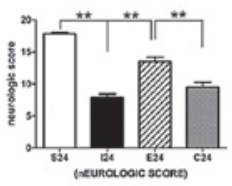

Figure 5. Effect of (-)-epigallocatechin-3-gallate (EGCG) on the protein expression of endoplasmic reticulum stress-related markers (A) GRP78, (B) CHOP and (c) caspase-12 in the ipsilateral cortex $24 \mathrm{~h}$ following reperfusion $(\mathrm{n}=3)$. (B) TdT mediated dUTP nick-end labeling (TUNEL) assay results ( $\mathrm{n}=3$ ). (C) Representative 2,3,5-triphenyltetrazolium chloride (TTC)-stained brain sections (n=6). (D) Neurology deficits score (n=6). Application of EGCG significantly reduced the infarct volumes and neurological deficit scores compared with middle cerebral artery occlusion (MCAO) group. Group S, sham-operated group; group I, MCAO group; group E, MCAO treated with EGCG group; group C, MCAO treated with EGCG and PD98059. Values are presented as the mean \pm SEM. ${ }^{*} \mathrm{P}<0.05$ and ${ }^{* *} \mathrm{P}<0.01$.

EGCG significantly reduces infarct volumes and promotes functional recovery in ipsilateral ischemic hemispheres $24 \mathrm{~h}$ following reperfusion. Following ischemia/reperfusion injury, a white-stained infarct area was observed in the MCAO group. By contrast, treatment with EGCG significantly reduced infarct volumes compared with that of the MCAO group $24 \mathrm{~h}$ following reperfusion $(\mathrm{P}<0.01)$. Following the application of PD98059, the infarct volumes were significantly increased compared with that of the EGCG-treated group $(\mathrm{P}<0.01$; Fig. 5E).

There was also significant improvement in the neurological score $24 \mathrm{~h}$ following reperfusion with EGCG treatment $(\mathrm{P}<0.01)$. When treated with PD98059, the neurological scores were significantly decreased compared with that of the EGCG-treated group $(\mathrm{P}<0.01$; Fig. 5F).

\section{Discussion}

The results clearly demonstrated that ICV injection of EGCG at low doses immediately following ischemia improved the outcome as measured by TTC staining and neurological scoring. EGCG treatment also significantly inhibited ERS and apoptosis. EGCG improved the neurological status and inhibited ERS, which was correlated with elevated TRPC6 and p-CREB activity and decreased SBDP145 activity. When MEK activity was inhibited, the neuroprotective effect of EGCG was attenuated and a correlated decrease in CREB activity was observed. These results demonstrated that
EGCG, is important in the prevention of cerebral ischemic injury (17), and may be used as a therapeutic intervention for stroke during the acute or subacute period similar to the drug edaravone (18).

Calpains are intracellular calcium-dependent cysteine endopeptidases that are activated by cytosolic $\mathrm{Ca}^{2+}$ overload (19). The most studied target of calpain is aII-spectrin, a $280-\mathrm{kDa}$ neuronal protein that localizes to axons and functions in cortical cytoskeleton matrix support. The aII-spectrin breakdown product, SBDP145, results from the sequential calpain cleavage of aII-spectrin which generates SBDP150 followed by cleavage to remove the additional $5 \mathrm{kDa}(20,21)$. In the present study, MCAO rats exhibited elevated levels of SBDP145 in the cortical regions of the ipsilateral hemisphere in the first $24 \mathrm{~h}$ following ischemic injury. EGCG treatment significantly reduced SBDP145 formation at 12 and $24 \mathrm{~h}$. The results clearly demonstrated that EGCG, when applied immediately following ischemia, inhibited calpain activation and induced resistance to ischemia/reperfusion injuries.

TRPC channels are non-selective cation channels that are expressed in numerous multicellular organisms with different functions (5). TRPC6 is involved in brain-derived neurotrophic factor (BDNF)-mediated growth cone turning, neuron survival and spine formation $(8,22)$. TRPC6 was specifically degraded in transient ischemia and this degradation occurred prior to and during neuronal cell death. In addition, TRPC6 protein in neurons in ischemia was specifically downregulated by calpain proteolysis. Inhibition of calpain proteolysis of 
TRPC6 protected animals from ischemic brain damage. In the present study, the protein levels of TRPC6 were markedly decreased at $6 \mathrm{~h}$ and the reduction in TRPC6 protein levels remained at 12 and $24 \mathrm{~h}$ in the MCAO group, these results were consistent with a previous study (7). EGCG treatment significantly enhanced the protein levels of TRPC6 at 12 and $24 \mathrm{~h}$. In addition, EGCG-treated rats exhibited significantly lower infarct volumes and also increased functional recovery compared with that of MCAO rats at $24 \mathrm{~h}$. Therefore, the results indicated that EGCG treatment protected rats from ischemic brain damage through the inhibition of calpain proteolysis of TRPC6.

A modest level of $\mathrm{Ca}^{2+}$ influx through TRPC6 channels leads to the activation of ERK, which activates CREB to promote neuronal survival (8). Inhibition of TRPC6 channel degradation maintained the phosphorylation of CREB and prevented ischemic brain damage (7). CREB activation is a critical event in the neuroprotection from ischemic injury $(23,24)$. In the present study, the protein levels of p-CREB were significantly increased in the EGCG-treated group at 12 and $24 \mathrm{~h}$. When MEK activity was inhibited, the neuroprotective effect of EGCG was attenuated and correlated with decreased CREB activity levels. The results demonstrated that EGCG, when administered immediately following ischemia, stimulated the MEK/ERK pathway that ultimately induced CREB activation and contributed to neuroprotection at $24 \mathrm{~h}$. In addition, EGCG significantly reduced TRPC6 degradation induced by ischemia at $24 \mathrm{~h}$. Therefore, the results suggested that EGCG blocked calpain-mediated TRPC6 channel degradation which activated CREB through the MEK/ERK pathway and contributed to neuroprotection.

The ER is an organelle that is important in the maintenance of intracellular calcium homeostasis and proper folding of newly synthesized secretory and membranous proteins (25). ER functions are disturbed by different insults, such as the accumulation of unfolded proteins and the disruption of intracellular calcium homeostasis $(26,27)$, which result in ER stress. However, if ER stress is too severe, the unfolded protein response initiates the apoptotic pathway (28). Increased expression of GRP78 is a marker of ER stress $(29,30)$. In addition, CHOP participates in apoptosis signaling pathways and serves as a hallmark of ER stress $(31,32)$. ER stress-induced neuronal cell death is important in stroke pathophysiology (33) and involves the activation of caspase-12 $(34,35)$, which is specific to apoptosis mediated by ER stress (9). In the present study, EGCG treatment significantly decreased the infarct volumes and improved functional recovery at $24 \mathrm{~h}$. In addition, the ER stress was enhanced in the brain following ischemia/reperfusion as demonstrated by the significant elevation of the ER stress-related markers GRP78, CHOP and caspase-12 in the cortex and EGCG was demonstrated to inhibit this. However, the effect of EGCG was attenuated by the MEK inhibitor PD98059. In addition, when treated with PD98059, the apoptotic cell death was also significantly increased. Thus, this study has demonstrated that ICV injection of EGCG inhibited ER stress and apoptosis through the MEK/ERK/CREB pathway, which contributed to its neuroprotective effects. The results indicated that EGCG exerted its neuroprotective effects by activating ERK/CREB pathways and the subsequent inhibition of ERS.
In conclusion, the present study has demonstrated that administration of EGCG immediately following ischemia inhibited ER stress and improved the neurological status through the inhibition of calpain proteolysis of TRPC6 and the subsequent activation of CREB via the MEK/ERK pathway.

\section{Acknowledgements}

This study was supported by the National Natural Science Foundation of China (grant no. 30901984).

\section{References}

1. Kaufman RJ: Stress signaling from the lumen of the endoplasmic reticulum: coordination of gene transcriptional and translational controls. Genes Dev 13: 1211-1233, 1999.

2. Zhang B, Rusciano D and Osborne NN: Orally administered epigallocatechin gallate attenuates retinal neuronal death in vivo and light-induced apoptosis in vitro. Brain Res 1198: 141-152, 2008.

3. Vivien D, Gauberti M, Montagne A, Defer G and Touzé E: Impact of tissue plasminogen activator on the neurovascular unit: from clinical data to experimental evidence. J Cereb Blood Flow Metab 31: 2119-2134, 2011

4. Martinou JC, Dubois-Dauphin M, Staple JK, et al: Overexpression of BCL-2 in transgenic mice protects neurons from naturally occurring cell death and experimental ischemia. Neuron 13: 1017-1030, 1994.

5. Montell C, Birnbaumer L and Flockerzi V: The TRP channels, a remarkably functional family. Cell 108: 595-598, 2002.

6. Harteneck C, Plant TD and Schultz G: From worm to man: three subfamilies of TRP channels. Trends Neurosci 23: 159-166, 2000.

7. Du W, Huang J, Yao H, Zhou K, Duan B and Wang Y: Inhibition of TRPC6 degradation suppresses ischemic brain damage in rats. J Clin Invest 120: 3480-3492, 2010.

8. Jia Y, Zhou J, Tai Y and Wang Y: TRPC channels promote cerebellar granule neuron survival. Nat Neurosci 10: 559-567, 2007.

9. Graham HN: Green tea composition, consumption, and polyphenol chemistry. Prev Med 21: 334-350, 1992.

10. Xie W, Ramakrishna N, Wieraszko A and Hwang YW: Promotion of neuronal plasticity by (-)-epigallocatechin-3-gallate. Neurochem Res 33: 776-783, 2008.

11. Haque AM, Hashimoto M, Katakura M, Tanabe Y, Hara Y and Shido O: Long-term administration of green tea catechins improves spatial cognition learning ability in rats. J Nutr 136: 1043-1047, 2006.

12. van Praag H, Lucero MJ, Yeo GW, et al: Plant-derived flavanol (-)epicatechin enhances angiogenesis and retention of spatial memory in mice. J Neurosci 27: 5869-5878, 2007.

13. Nagai K, Jiang MH, Hada J, et al: (-)-Epigallocatechin gallate protects against NO stress-induced neuronal damage after ischemia by acting as an anti-oxidant. Brain Res 956: 319-322, 2002.

14. Sutherland BA, Shaw OM, Clarkson AN, Jackson DN, Sammut IA and Appleton I: Neuroprotective effects of (-)-epigallocatechin gallate following hypoxia-ischemia-induced brain damage: novel mechanisms of action. FASEB J 19: 258-260, 2005.

15. Garcia JH, Wagner S, Liu KF and Hu XJ: Neurological deficit and extent of neuronal necrosis attributable to middle cerebral artery occlusion in rats. Statistical validation. Stroke 26: 627-635, 1995.

16. Tsubokawa T, Jadhav V, Solaroglu I, Shiokawa Y, Konishi Y and Zhang JH: Lecithinized superoxide dismutase improves outcomes and attenuates focal cerebral ischemic injury via antiapoptotic mechanisms in rats. Stroke 38: 1057-1062, 2007.

17. Arab L, Liu W and Elashoff D: Green and black tea consumption and risk of stroke: a meta-analysis. Stroke 40: 1786-1792, 2009.

18. Zhang N, Komine-Kobayashi M, Tanaka R, Liu M, Mizuno Y and Urabe T: Edaravone reduces early accumulation of oxidative products and sequential inflammatory responses after transient focal ischemia in mice brain. Stroke 36: 2220-2225, 2005.

19. Goll DE, Thompson VF, Li H, Wei W and Cong J: The calpain system. Physiol Rev 83: 731-801, 2003. 
20. Nath R, Raser KJ, Stafford D, et al: Non-erythroid alpha-spectrin breakdown by calpain and interleukin 1 beta-converting-enzyme-like protease(s) in apoptotic cells: contributory roles of both protease families in neuronal apoptosis. Biochem J 319: 683-690, 1996.

21. Wang KK: Calpain and caspase: can you tell the difference?, by kevin K.W. WangVol. 23, pp. 20-26. Trends Neurosci 23: 59, 2000.

22. Li Y, Jia YC, Cui K, et al: Essential role of TRPC channels in the guidance of nerve growth cones by brain-derived neurotrophic factor. Nature 434: 894-898, 2005

23. Walton MR and Dragunow I: Is CREB a key to neuronal survival? Trends Neurosci 23: 48-53, 2000.

24. Finkbeiner S: CREB couples neurotrophin signals to survival messages. Neuron 25: 11-14, 2000

25. Yin XM, Oltvai ZN and Korsmeyer SJ: BH1 and BH2 domains of Bcl-2 are required for inhibition of apoptosis and heterodimerization with Bax. Nature 369: 321-323, 1994.

26. He B: Viruses, endoplasmic reticulum stress, and interferon responses. Cell Death Differ 13: 393-403, 2006.

27. Ginsberg MD: Neuroprotection for ischemic stroke: past, present and future. Neuropharmacology 55: 363-389, 2008.

28. Love S: Apoptosis and brain ischaemia. Prog Neuropsychopharmacol Biol Psychiatry 27: 267-282, 2003.
29. Yoshida H, Matsui T, Yamamoto A, Okada T and Mori K: XBP1 mRNA is induced by ATF6 and spliced by IRE1 in response to ER stress to produce a highly active transcription factor. Cell 107: 881-891, 2001

30. Groenendyk J, Sreenivasaiah PK, Kim do H, Agellon LB and Michalak M: Biology of endoplasmic reticulum stress in the heart. Circ Res 107: 1185-1197, 2010.

31. DeGracia DJ and Montie HL: Cerebral ischemia and the unfolded protein response. J Neurochem 91: 1-8, 2004.

32. Tajiri S, Oyadomari S, Yano S, et al: Ischemia-induced neuronal cell death is mediated by the endoplasmic reticulum stress pathway involving CHOP. Cell Death Differ 11: 403-415, 2004.

33. Zhu DY, Lau L, Liu SH, Wei JS and Lu YM: Activation of cAMP-response-element-binding protein (CREB) after focal cerebral ischemia stimulates neurogenesis in the adult dentate gyrus. Proc Natl Acad Sci USA 101: 9453-9457, 2004.

34. Zinkstok SM, Vergouwen MD and Engelter ST, et al: Safety and functional outcome of thrombolysis in dissection-related ischemic stroke: a meta-analysis of individual patient data. Stroke 42: 2515-2520, 2011.

35. Paschen W and Mengesdorf T: Cellular abnormalities linked to endoplasmic reticulum dysfunction in cerebrovascular disease - therapeutic potential. Pharmacol Ther 108: 362-375, 2005. 